\title{
Synergy between early-incorporation immunotherapy and extracranial radiotherapy in metastatic non-small cell lung cancer
}

\author{
Parth A. Chodavadia ${ }^{1 \#}$, Corbin D. Jacobs ${ }^{2 \#}$, Frances Wang ${ }^{3}$, Joseph K. Salama ${ }^{2}$, Chris R. Kelsey ${ }^{2}$, \\ Jeffrey M. Clarke ${ }^{4}$, Neal E. Ready ${ }^{4}$ Jordan A. Torok ${ }^{2}$ \\ ${ }^{1}$ School of Medicine, Duke University, Durham, NC 27710, USA; ${ }^{2}$ Department of Radiation Oncology, Duke University, Durham, NC 27710, USA; \\ ${ }^{3}$ Department of Biostatistics and Bioinformatics, Duke University, Durham, NC 27710, USA; ${ }^{4}$ Duke Cancer Institute, Duke University Medical \\ Center, Durham, NC 27710, USA \\ Contributions: (I) Conception and design: PA Chodavadia, CD Jacobs; (II) Administrative support: PA Chodavadia, CD Jacobs; (III) Provision of study \\ materials or patients: PA Chodavadia, CD Jacobs; (IV) Collection and assembly of data: PA Chodavadia, CD Jacobs, F Wang; (V) Data analysis and \\ interpretation: PA Chodavadia, CD Jacobs, F Wang, NE Ready, JA Torok; (VI) Manuscript writing: All authors; (VII) Final approval of manuscript: \\ All authors. \\ \#These authors contributed equally to this work. \\ Correspondence to: Joseph K. Salama. Department of Radiation Oncology, Box 3085, Duke University Medical Center, Durham, NC 27710, USA. \\ Email: joseph.salama@duke.edu.
}

Background: Combining radiotherapy (RT) and immunotherapy (IT) may enhance outcomes for metastatic non-small cell lung cancer (mNSCLC). However, data on the immunomodulatory effects of extracranial RT remains limited. This retrospective database analysis examined real-world practice patterns, predictors of survival, and comparative effectiveness of extracranial radioimmunotherapy (RT + IT) versus early-incorporation immunotherapy (eIT) in patients with mNSCLC.

Methods: Patients diagnosed with mNSCLC between 2004-2016 treated with eIT or RT + IT were identified in the National Cancer Database. Practice patterns were assessed using Cochrane-Armitrage trend test. Cox proportional hazards and Kaplan-Meier method were used to analyze overall survival (OS). Propensity score matching was performed to account for baseline imbalances. Biologically effective doses (BED) were stratified based on the median $\left(39 \mathrm{~Gy}_{10}\right)$. Stereotactic body radiotherapy (SBRT) was defined as above median BED in $\leq 5$ fractions.

Results: eIT utilization increased from $0.3 \%$ in 2010 to $13.2 \%$ in 2016 ( $\mathrm{P}<0.0001)$. Rates of RT + eIT increased from $38.8 \%$ in 2010 to $49.1 \%$ in 2016 among those who received eIT $(\mathrm{P}<0.0001)$. Compared to eIT alone, RT + eIT demonstrated worse median OS (11.2 vs. 13.2 months) while SBRT + eIT demonstrated improved median OS (25 vs. 13.2 months) $(\mathrm{P}<0.0001)$. There were no significant differences in OS based on sequencing of eIT relative to RT (log-rank $\mathrm{P}=0.4415)$ or irradiated site (log-rank $\mathrm{P}=0.1606)$. On multivariate analysis, factors associated with improved OS included chemotherapy (HR 0.86, P=0.0058), treatment at academic facilities (HR 0.83, $\mathrm{P}<0.0001$ ), and SBRT (HR 0.60, $\mathrm{P}=0.0009$ ); after propensity-score multivariate analysis, SBRT alone showed improved OS (HR 0.28, $\mathrm{P}<0.0001)$.

Conclusions: Utilization of RT + eIT in mNSCLC is increasing. SBRT + eIT was associated with improved OS on propensity-score matched analysis. There were no significant differences in OS based on RT + eIT sequencing or site irradiated. Whether these observations reflect patient selection or possible immunomodulatory benefits of RT is unclear and warrants further study.

Keywords: Database analysis; immunotherapy; non-small cell lung cancer (NSCLC); radioimmunotherapy; radiotherapy

Submitted Apr 07, 2020. Accepted for publication Nov 01, 2020.

doi: $10.21037 /$ tlcr-20-537

View this article at: http://dx.doi.org/10.21037/tlcr-20-537 


\section{Introduction}

Metastatic non-small cell lung cancers (mNSCLC) account for more than $80 \%$ of all lung cancers in the United States (1). Most NSCLC patients are diagnosed with distant metastases, resulting in poor 5 -year overall survival (OS) (2). Beginning with pembrolizumab in 2017, several immunotherapy (IT) drugs have been approved for first-line treatment for mNSCLC based on significantly improved OS (3-5). However, there is still room for significant improvement, and there is significant interest in developing new combination therapeutic strategies to continue to improve outcomes for mNSCLC patients. In particular, there are a number of current investigations looking at interaction of radiotherapy (RT) and systemic therapies that modulate the host immune system (6).

Emerging preclinical evidence suggests that RT in combination with IT may drive immunomodulation in the local tumor microenvironment (7-10). A secondary analysis of patients with mNSCLC enrolled on KEYNOTE-001 demonstrated that those who received extra-cranial RT prior to pembrolizumab administration had significantly higher OS and progression free survival (PFS) than patients who did not receive prior RT (11). In addition, combining chemoradiotherapy with IT does not appear to significantly increase toxicities in stage IIIB and IV NSCLC (12-14).

Despite these results, there is lack of clinical data to directly support synergy of RT and IT (15). A single prior study analyzing the National Cancer Database (NCDB) reported that stereotactic $\mathrm{RT}(>80 \%$ intracranial) was associated with improved OS among patients receiving IT, whereas non-stereotactic RT was associated with reduced OS (16). However, this conclusion could be attributable to patient selection bias and not necessarily differences in immunomodulation based on RT. To date, no study has examined practice patterns of RT utilization in mNSCLC patients that receive early-incorporation IT (eIT), defined as IT receipt within 120 days of diagnosis. This is especially important as more IT regimens gain approval as firstline treatment for mNSCLC. In addition, differences in immunomodulation from varying aspects of extracranial RT, such as sequencing, site irradiated, and dose-fractionation, are unknown.

Our primary objective was to analyze practice patterns of eIT and utilization of RT in patients with mNSCLC using the NCDB. Secondary objectives were to (A) identify predictors of OS in mNSCLC patients who received eIT with or without extracranial RT and (B) perform a hypothesis-generating comparative effectiveness analysis in patients that received eIT and extracranial RT based on RT + eIT sequencing, irradiated site, and RT dosefractionation. We hypothesized that an increasing number of patients received RT + eIT since 2004 and that patients who received RT + eIT had improved OS compared to eIT alone. We present the following article in accordance with the STROBE guideline checklist (available at http://dx.doi. org/10.21037/tlcr-20-537).

\section{Methods}

In this study, we conducted a retrospective cohort database analysis with a specific focus on patients with mNSCLC who received eIT or RT + eIT from 2004 to 2016 included in the NCDB. The NCDB is a joint project of the Commission on Cancer of the American College of Surgeons and the American Cancer Society that captures approximately $70 \%$ of newly diagnosed malignancies in the United States (17). Hospitals accredited by the Commission on Cancer provide data to the NCDB from their registries using standardized coding and data item definitions (18). We used the NCDB because other databases like the Surveillance, Epidemiology, and End Results (SEER) do not distinguish between chemotherapy and IT (18).

De-identified data for patients ages 18 to 90 diagnosed with histologically-confirmed lung cancers from 2004 to 2016 were obtained from the NCDB Participant User File. Patients were excluded from the patterns-of-care analysis for the following reasons: no distant metastases, American Joint Committee on Cancer (AJCC) $7^{\text {th }}$ edition stage I-III tumors, diagnosis of prior malignancies, or nonNSCLC histologies (e.g., small cell, carcinoid, or sarcoma). Additionally, patients were excluded from the comparative effectiveness analysis for the following reasons: no IT delivery, IT administered $>120$ days after the diagnosis date (i.e., above a pre-defined early-incorporation threshold), prolonged radiation duration ( $>60$ days), intracranial or inappropriate (e.g., prostate or whole body) radiation site, brachytherapy modality, and missing survival status. Patients with pleural or pericardial metastases (AJCC $8^{\text {th }}$ edition M1a) as the only identifiable reason for metastatic disease were also excluded since these patients have more favorable prognosis with similar survival to stage IIIC NSCLC and typically do not receive palliative RT (19).

Cumulative RT dose in Gray (Gy) and the number of RT fractions were used to calculate the biologically effective dose (BED) using the formula $B E D=n * d *(1+d \div[\alpha / \beta])$ 
where $\mathrm{n}=$ number of fractions, $\mathrm{d}=$ dose per fraction, and 10 is the assumed alpha/beta ratio for NSCLC tumors $(20,21)$. With the exception of 8 Gy in 1 fraction (BED=14.4 $G_{10}$ ), 10 Gy in 1 fraction $\left(B E D=20.0 G_{10}\right), 20$ Gy in 5 fractions (BED=28 $\left.\mathrm{Gy}_{10}\right), 17$ Gy in 2 fractions (BED=31.45 $\mathrm{Gy}_{10}$ ), and 24 Gy in 6 fractions $\left(\mathrm{BED}=33.5 \mathrm{~Gy}_{10}\right)$, which are five commonly used palliative dose-fractionations endorsed by consensus American Society of Radiation Oncology guidelines (22), patients with other low BED values $<39 \mathrm{~Gy}_{10}$ were excluded (23). The upper limit of acceptable BED was based on 60 Gy in 3 fractions, and all other BED values $>180 \mathrm{~Gy}_{10}$ were excluded. Stringent inclusion criteria were applied because anomalous RT data has been shown to significantly alter survival results (24). All remaining patients were then either assigned to the RT + eIT cohort if their first coded course of RT was to an extracranial site or to the eIT only cohort. A limited number of patients with brain metastases at diagnosis not treated with intracranial irradiation as first course, potentially because they were small and/or asymptomatic, were included in the eIT only cohort to minimize bias.

Three subgroups were created within the RT + eIT cohort based on the biologically effective radiation dose (BED), treatment sequencing, and irradiated site. All patients who received RT + eIT were stratified into three categories: $\mathrm{BED}<39, \mathrm{BED}=39$, and $\mathrm{BED}>39$. BED $=39 \mathrm{~Gy}_{10}$ was used as a cutoff because it represented the median and comprised nearly half of the entire population of patients that received RT. Patients in the highest BED stratum treated in 1-5 fractions were categorized as receiving stereotactic body radiotherapy (SBRT) (25) to account for patients treated aggressively and who may have had oligometastatic disease. Treatment sequencing subgroups were created based on whether the patient received eIT after completing RT or whether eIT was administered before or during RT. Irradiated site subgroups were created based on whether the patient received their first course of RT to the lung/mediastinum, an osseous site, or other site. Due to insufficient power and limitations in the data dictionary, the 'osseous' subgroup (e.g., spine, rib, pelvis, femur, humerus, etc.) and the 'other' subgroup (e.g., liver, adrenal, lymph node) was not further subdivided.

\section{Statistical analysis}

Demographic and clinical covariates for analysis included mean age at diagnosis, race, sex, insurance status, facility type, Charlson-Deyo Comorbidity Index (26), histology, grade, number of organs with metastases, clinical $\mathrm{N}$ and $\mathrm{T}$ stages, and receipt of CT. Chi-squared test and $t$-tests were used to analyze differences in baseline characteristics between patients who received RT + eIT versus RT alone. Patterns-of-care analyses examined three trends between 2010 and 2016: (I) eIT among all patients with mNSCLC, (II) RT utilization among patients with mNSCLC who received eIT, and (III) sequencing of eIT and RT among patients with mNSCLC who received RT + eIT. The expected proportion of individuals receiving a specific treatment regimen and $95 \%$ confidence interval (CI) were also calculated using bivariate logistic regression with year of diagnosis as a nominal variable. The CochraneArmitrage test was conducted to evaluate trends from 2010 to 2016.

Overall survival was calculated from diagnosis to death (event data) or last date of follow-up (censored data). Predictors of overall survival (OS) were compared among patients with eIT using Cox proportional hazards regression model, providing hazard ratios (HR) with $95 \%$ CI. All variables were included in a logistic regression model to calculate HRs. Kaplan-Meier methods were used for univariate survival analyses while the log-rank test was used to make OS comparisons between and/or among groups based on RT receipt, sequencing of eIT relative to RT, and BED. 1:1 matching using the nearest neighbor algorithm was performed with patients who received no RT and those that received $\mathrm{BED}>39 \mathrm{~Gy}_{10}$ (excluding SBRT) to calculate propensity-matched scores. Patients were matched based on diagnosis year, age, race, sex, comorbidity index, insurance status, facility type, histology, number of organs with metastasis, and chemotherapy receipt. A Wald chi-square interaction test was also conducted to assess the relationship between BED and sequencing of eIT relative to RT. Any patient with missing data was excluded from the overall survival analysis. Given that the NCDB is a centralized database, we do not anticipate that a certain type of patient is more likely to have missing data than others, mitigating risk for bias.

Statistical analyses were performed using SAS version 9.4 (SAS Institute Incorporated, Cary, NC). A P value less than 0.05 was considered significant for all analyses. The Institutional Review Board deemed this study exempt from review given the deidentified nature of the data. All procedures performed in this study were in accordance with the Declaration of Helsinki (as revised in 2013). Because of the retrospective nature of the research, the requirement for informed consent was waived. 


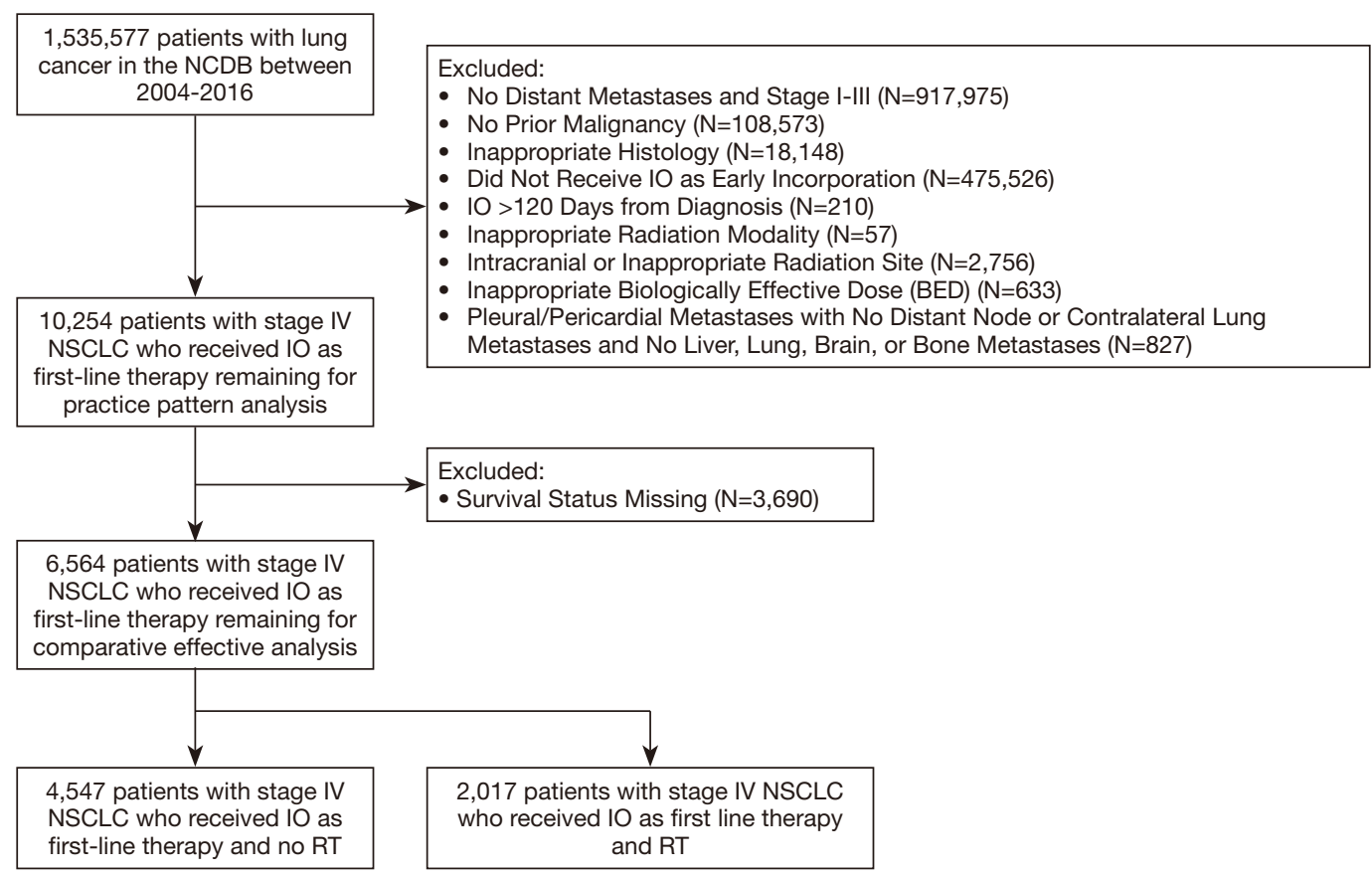

Figure 1 CONSORT diagram. NCDB, national cancer database; IO, immunotherapy; RT, radiation therapy; NSCLC, non-small cell lung cancer.

\section{Results}

Between 2004 and 2016, 490,701 patients diagnosed with mNSCLC were included in the NCDB (Figure 1). Of these, 14,301 patients received eIT between 2010 and 2016, increasing from $0.3 \%$ in 2010 to $13.2 \%$ in 2016 $(\mathrm{P}<0.0001)$. Among patients that received eIT, 6,687 (46.8\%) also received RT, increasing from $38.8 \%$ in 2010 to $49.0 \%$ in $2016(\mathrm{P}<0.0001)$ (Figure $2 A)$. In particular, there was an increase in the proportion of patients receiving SBRT from $8.5 \%$ in 2010 to $18.7 \%$ in 2016 with a corresponding decrease in the proportion of patients receiving $\mathrm{BED}>39 \mathrm{~Gy}_{10}$ from $66.0 \%$ in 2010 to $24.1 \%$ in 2016 (Figure 2B). 4,188 (67.4\%) patients received eIT after completion of their first RT course, increasing from $46.7 \%$ in 2010 to $67.4 \%$ in $2016(\mathrm{P}=0.0001)$. Bivariate logistic regression analysis with $95 \%$ CIs further confirmed that the proportion of patients receiving $\mathrm{RT}+\mathrm{IT}$ irrespective of sequencing $(\mathrm{P}<0.0001)$ and eIT after RT $(\mathrm{P}=0.0005)$ significantly increased from 2010 to 2016 despite marked differences in sample size across years.

\section{Baseline characteristics analysis}

A total of 6,564 patients were included in the overall survival analysis. Of these, 4,547 (69.3\%) were treated without RT and 2,017 (31.7\%) received RT. Baseline characteristics are shown in Table 1.

There were significant baseline imbalances between patients who received RT versus those that did not receive RT. Patients who received RT were more likely to be slightly younger at diagnosis $(\mathrm{P}<0.0001)$, have private insurance $(\mathrm{P}<0.0001)$, and receive care outside an academic or comprehensive care center $(\mathrm{P}=0.0009)$. Patients who received RT had a higher proportion of nonadenocarcinoma histology $(\mathrm{P}<0.0001)$ and metastases in more organs at diagnosis $(\mathrm{P}<0.0001)$.

Patients who received RT were also stratified by BED. Two hundred eighty-three (14.0\%) patients received BED $<39 \mathrm{~Gy}_{10}, 917$ (45.5\%) patients received $\mathrm{BED}=39 \mathrm{~Gy}_{10}$, and $817(40.5 \%)$ patients received BED >39 Gy $\mathrm{G}_{10}$. Patients who received $\mathrm{BED}=39 \mathrm{~Gy}_{10}$ most commonly received a dose-fractionation of $30 \mathrm{~Gy}$ in 10 fractions ( $99 \%)$. Of the patients who received $\mathrm{BED}>39 \mathrm{~Gy}_{10}, 122$ patients (14.9\%) received SBRT. The most common dose-fractionations in the SBRT cohort were 30 Gy in 5 fractions (17\%), 50 Gy in 5 fractions (14\%), 27 Gy in 3 fractions (9\%), 24 Gy in 3 fractions (7\%), 40 Gy in 5 fractions (7\%), and 30 Gy in 3 fractions (7\%). Patients with BED $<39 \mathrm{~Gy}_{10}$ were less likely to receive chemotherapy $(\mathrm{P}<0.0001)$ and had more organs involved with metastases at diagnosis $(\mathrm{P}<0.0001)$. 

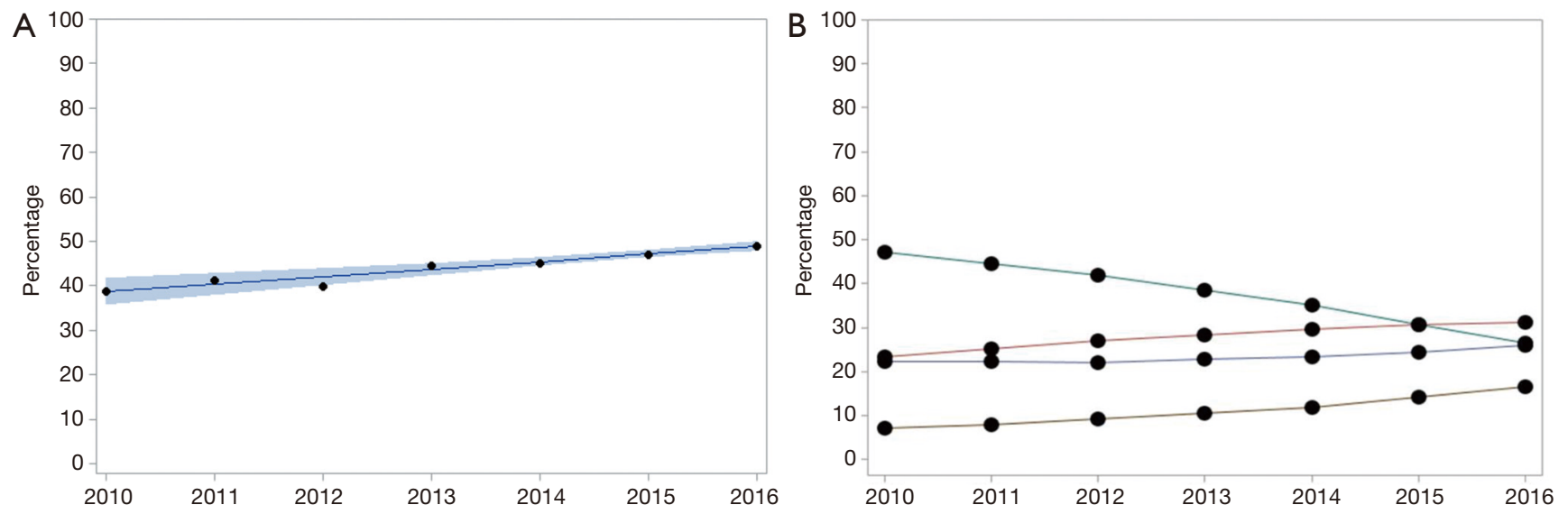

Figure 2 Patterns of care analysis, 2010-2016. (A) Utilization of eIT and RT in patients with mNSCLC increased from $38.8 \%$ in 2010 to 49.0\% in 2016 ( $\mathrm{N}=6,687, \mathrm{P}<0.0001)$. (B) Utilization SBRT (brown) increased from $7.2 \%$ in 2010 to $16.5 \%$ in 2016 . Trends for BED <39 (blue), BED =39 (red), and BED >39 (green) are also shown. eIT, early-incorporation immunotherapy; RT, radiotherapy; NSCLC, nonsmall cell lung cancer.

\section{Kaplan-Meier survival analysis}

Kaplan-Meier plots investigating the relationship of RT + eIT versus eIT alone are shown in Figure $3 A$. Patients who received RT + eIT (median OS, 11.2 months) demonstrated worse OS compared to patients who received eIT alone (median OS, 13.2 months) (log-rank $\mathrm{P}<0.0001$ ).

Within the cohort that received RT + eIT, there were no significant differences in OS for patients receiving eIT after RT or eIT before or concomitant to RT (logrank $\mathrm{P}=0.4333$ ). Dose-dependent stratification revealed significant differences in OS, with OS increasing with higher BED doses (log-rank $\mathrm{P}<0.0001$; Figure $3 B$ ). Median OS was 25.0, 12.6, 10.8, and 8.3 months for SBRT, BED $>39 \mathrm{~Gy}_{10}, \mathrm{BED}=39 \mathrm{~Gy}_{10}$, and $\mathrm{BED}<39 \mathrm{~Gy}_{10}$, respectively. Patients treated with SBRT and eIT had improved OS compared to patients who received eIT alone (median OS 25.0 versus 13.2 months, $\log$-rank $\mathrm{P}<0.0001$ ). There were no significant differences in OS based on irradiated site (lung versus osseous versus other targets) (log-rank $\mathrm{P}=0.1395)$.

A significant interaction was observed between BED and sequencing of eIT relative to RT (Wald chi-square test, $\mathrm{P}=0.005$ for bivariate and $\mathrm{P}=0.02$ for adjusted). Results of this analysis are shown in Table 2. Patients who received $\mathrm{BED}<39 \mathrm{~Gy}_{10}(\mathrm{P}=0.0037)$ or $\mathrm{BED}=39 \mathrm{~Gy}_{10}(\mathrm{P}=0.0185)$ had improved OS when eIT was delivered after RT compared to patients who received eIT before or concomitant to RT. BED $>39 \mathrm{~Gy}_{10}$ and SBRT did not show significant OS differences based on eIT sequencing relative to RT. Given the variability in timing of eIT and RT within each cohort (Table 3), we also conducted a sensitivity analysis restricting the number of days between eIT and RT to 30 days (results not shown). This analysis corroborated prior findings that patients who received BED $<39 \mathrm{~Gy}_{10}$ (HR 0.69, 95\% CI: 0.49-0.97) or BED =39 Gy Gy $_{10}$ (HR 86 , 95\% CI: 0.71-1.04) had improved OS when eIT was delivered after RT with no significant improvements in OOS for patients who received BED > 39 or SBRT.

\section{Predictors of overall survival analysis}

Bivariate and multivariate Cox proportional hazards regression model revealed that $\mathrm{RT}+\mathrm{eIT}$ overall was associated with worse survival (HR 1.13, 95\% CI: 1.061.20, $\mathrm{P}<0.0001)$ compared to eIT alone. A subsequent Cox proportional hazards regression model with dose stratifications by BED was conducted. Bivariate, multivariate, and propensity-score matched results are reported in Table 4. Factors significantly associated with higher risk of death on results from a multivariate model with 6,471 patients include: older age at diagnosis (HR 1.01, $\mathrm{P}=0.0008$ ), male sex (HR 1.24, $\mathrm{P}<0.0001$ ), Medicaid insurance (HR 1.23, $\mathrm{P}=0.0004)$, large cell (HR 1.51, $\mathrm{P}<0.0001$ ), non-small cell not otherwise specified (HR 1.31, $\mathrm{P}<0.0001$ ), squamous cell (HR 1.18, $\mathrm{P}=0.0017)$, $>1$ organ with metastases (HR 1.20, $\mathrm{P}<0.0001$ ), BED $<39$ $\mathrm{Gy}_{10}$ (HR 1.67, $\mathrm{P}<0.0001$ ), and BED $=39 \mathrm{~Gy}_{10}$ (HR 1.20, $\mathrm{P}<0.0001)$. Factors significantly associated with lower risk 
Table 1 Baseline demographic and tumor characteristics

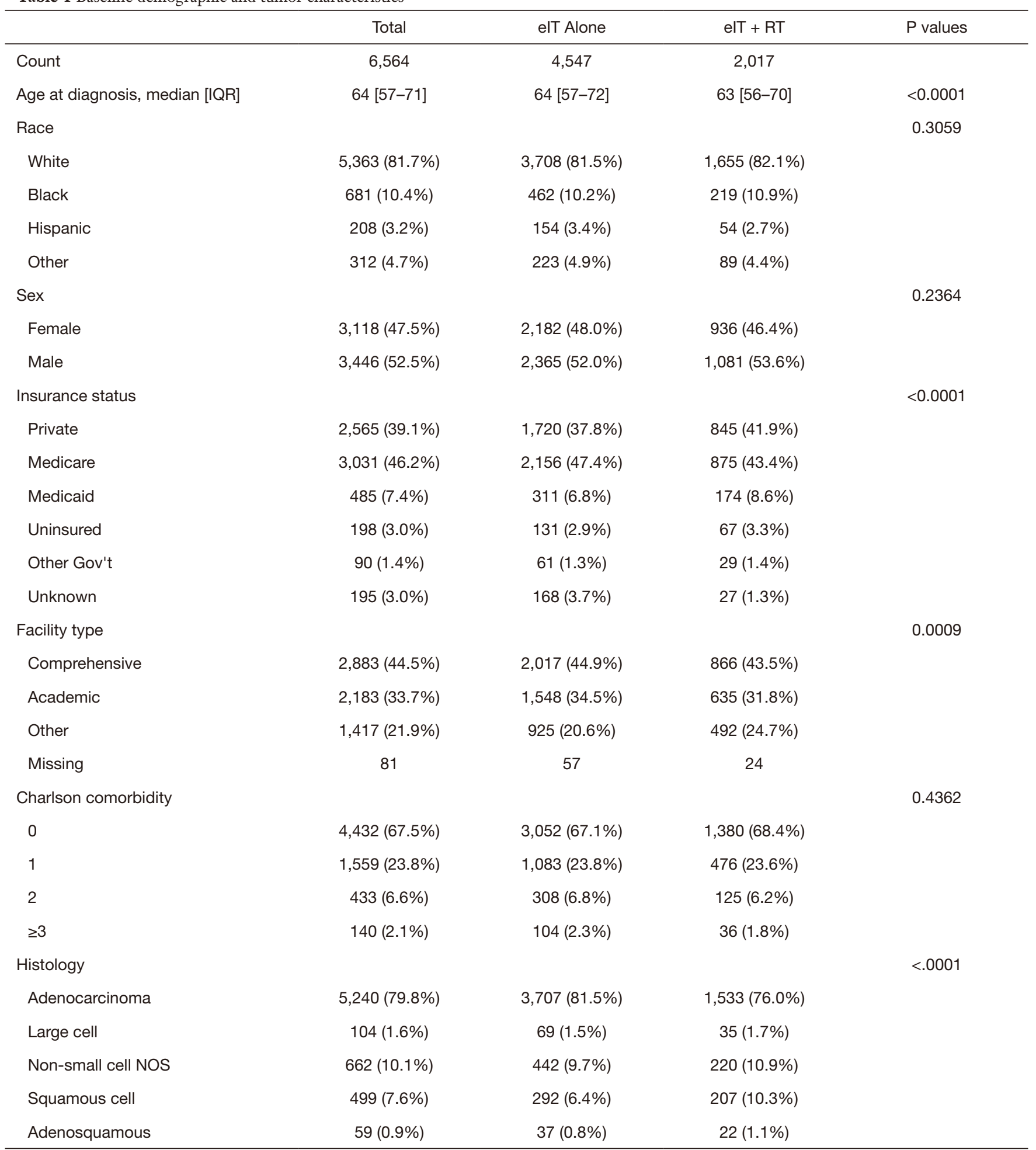

Table 1 (continued) 
Table 1 (continued)

\begin{tabular}{lccc}
\hline & Total & elT Alone & elT + RT \\
\hline Number of organs with metastasis & & & \\
0 & $2,599(39.6 \%)$ & $1,924(42.3 \%)$ & $675(33.5 \%)$ \\
1 & $2,699(41.1 \%)$ & $1,835(40.4 \%)$ & $864(42.9 \%)$ \\
2 & $1,034(15.7 \%)$ & $642(14.1 \%)$ & $392(19.4 \%)$ \\
3 & $212(3.2 \%)$ & $138(3.0 \%)$ & $74(3.7 \%)$ \\
4 & $20(0.3 \%)$ & $8(0.2 \%)$ & $12(0.6 \%)$ \\
Chemotherapy & & & 0.4117 \\
Yes & $6,056(92.4 \%)$ & $4,187(92.3 \%)$ & $1,869(92.8 \%)$ \\
No & $495(7.6 \%)$ & $351(7.7 \%)$ & $144(7.2 \%)$ \\
Missing & 13 & 9 & 4 \\
\hline
\end{tabular}

$\mathrm{RT}$, radiotherapy; elT, early-incorporation immunotherapy.

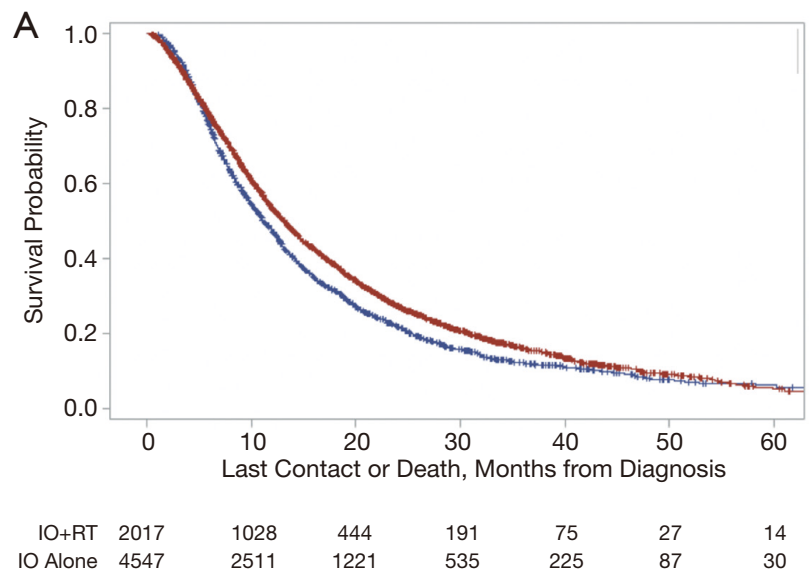

Figure 3 Kaplan-Meier regression analysis. (A) Survival analysis comparing patients who received radiotherapy and immunotherapy (blue) with immunotherapy alone (red) (log-rank test, $\mathrm{P}<0.0001)$. (B) Survival analysis comparing patients who received radiotherapy stratified by BED $<39$ (green), BED =39 (blue), BED > 39 (red), and SBRT (brown) (log-rank test, $\mathrm{P}<0.0001$ ). BED, biologically effective dose.

of death included: academic facility (HR $0.83, \mathrm{P}<0.0001$ ), chemotherapy receipt (HR 0.86, $\mathrm{P}=0.0051$ ), and SBRT (HR $0.58, \mathrm{P}=0.0003)$. After propensity-score matching, BED $>39 \mathrm{~Gy}_{10}$ was not associated with improved overall survival (HR 0.89, $\mathrm{P}=0.1526$ ). When a propensity-score matched analysis was conducted with SBRT (not shown), SBRT was associated with improved survival (HR $0.28, \mathrm{P}<0.0001)$.

\section{Discussion}

In this study we sought to (A) characterize patterns of care

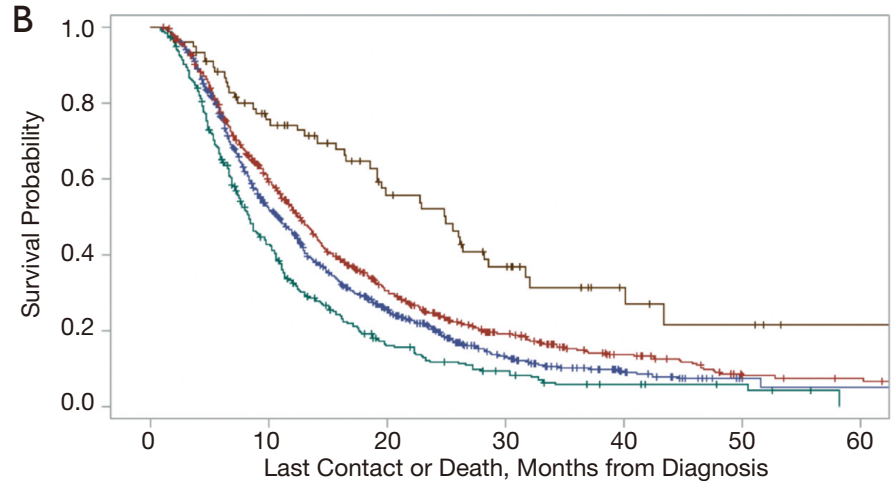

$\begin{array}{rlcccccc}\mathrm{BED}<39 & 283 & 111 & 35 & 17 & 7 & 4 & 0 \\ \mathrm{BED}=39 & 917 & 451 & 190 & 67 & 22 & 4 & 2 \\ \mathrm{BED}>39 & 740 & 414 & 188 & 89 & 39 & 15 & 11 \\ \mathrm{SBRT} & 77 & 52 & 31 & 18 & 7 & 4 & 1\end{array}$


Table 2 Interaction analysis between BED and RT + eIT sequencing

\begin{tabular}{lcc}
\hline & $\begin{array}{c}\text { elT Before or concomitant } \\
\text { to RT }(\mathrm{N}=817)\end{array}$ & $\begin{array}{c}\text { elT after RT } \\
(\mathrm{N}=1,153)\end{array}$ \\
\hline Median OS (months) & 6.83 & 9.20 \\
BED $<39$ & 9.63 & 11.96 \\
BED =39 & 13.47 & 12.06 \\
BED $>39$ (excl SRT) & 24.87 & 25.00 \\
SRT & & \\
HR $(95 \%$ Cl) & Ref & $0.68(0.52-0.88)$ \\
BED $<39$ & Ref & $0.83(0.72-0.97)$ \\
BED $=39$ & Ref & $1.07(0.91-1.26)$ \\
BED $>39$ (excl SRT) & Ref & $0.90(0.49-1.66)$ \\
SRT &
\end{tabular}

BED, biologically effective dose; RT, radiotherapy; elT, earlyincorporation immunotherapy.

Table 3 Number of days between RT and eIT by cohort

\begin{tabular}{lcc}
\hline & $\begin{array}{c}\text { elT Before or concomitant } \\
\text { to RT }(\mathrm{N}=817)\end{array}$ & $\begin{array}{c}\text { elT after RT } \\
(\mathrm{N}=1,153)\end{array}$ \\
\hline Minimum & 0 & 1 \\
First quartile & 13 & 9 \\
Median & 32 & 19 \\
Third quartile & 92 & 41 \\
Maximum & 783 & 1035 \\
\hline
\end{tabular}

$\mathrm{RT}$, radiotherapy; elT, early-incorporation immunotherapy.

of mNSCLC patients who received RT + eIT.

Contrary to our initial hypothesis, patients who received RT + eIT had worse OS than patients who received eIT alone. This is in contrast to results from PEMBRO-RT and KEYNOTE-001 $(11,15)$. PEMBRO-RT showed that patients with advanced NSCLC who received SBRT prior to pembrolizumab administration had a higher median PFS (6.6 months) compared to patients who received pembrolizumab alone (1.9 months) (15). In a secondary analysis of KEYNOTE-001, Shaverdian et al. also showed improved PFS (HR 0.56, 95\% CI: 0.34-0.91) and OS (HR $0.58,95 \%$ CI: $0.36-0.94)$ in patients who received any RT before the first cycle of pembrolizumab (11).

Our data may differ from these studies due to baseline imbalances among patients who received eIT alone $v$. combined RT + eIT. Palliative-intent RT is often utilized in patients with more advanced disease causing symptoms, thus these patients overall may be expected to have a worse prognosis. Patients in the KEYNOTE-001 secondary analysis included those who received prior RT with curative intent for stage I-III NSCLC at initial diagnosis. The RT subgroup also had significantly longer intervals between diagnosis and receipt of pembrolizumab, thus the favorable outcomes may have been a reflection of a more indolent biology and lead time bias. Conversely, patients included in our analysis received RT in the context of metastatic disease. In addition, patients who received combined RT + eIT were more likely to have non-adenocarcinoma histologies $(24.0 \%$ vs. $18.5 \%)$ and multiple organs with metastases $(23.7 \%$ vs. $17.3 \%)$ compared to those who received eIT alone. Adenocarcinoma histology and decreased metastatic burden are associated with better OS in NSCLC $(8,9,27)$. These factors collectively may explain the inferior outcomes overall. Randomization as done in PEMBRO-RT (NCT03396471) would be necessary to account for these imbalances.

Dose stratification revealed that SBRT was associated with improved OS on initial multivariate analysis and after propensity score matching. This is in line with Hasselle et al.'s findings that patients with oligometastatic NSCLC treated with hypofractionated image-guided radiotherapy had improved PFS (28). Multiple studies have shown that patients who receive SBRT in the setting of mNSCLC have improved OS and PFS compared to patients who received eIT alone (29-32). A recent database analysis also found that SBRT+IT was associated with improved OS compared to IT alone and external beam radiotherapy (EBRT) + IT in mNSCLC (16). SBRT is hypothesized to help release neoantigens, leading to maturation and proliferation of naïve T-cells, while immunotherapy activates and amplifies naïve T-cells (33). Both may also reciprocally potentiate each other's effects through further amplification of tumoricidal effects of T-cells (33). It is interesting to note that this study found improved OS even at palliative dosing given that many clinical studies examining the effects of SBRT on OS use ablative dosing. In fact, Mazzola et al. suggest that the effects of SBRT on immunomodulation is highly drugdependent and tissue-dependent (34). More robust data on dose escalation effects in mNSCLC patients is needed to evaluate whether these findings reflect patient selection or actually improved outcomes.

Lastly, we found no OS difference based on the sequencing of RT and eIT in the mNSCLC setting. There is a paucity of literature to guide the sequencing of radioimmunotherapy $(11,12)$. A subgroup analysis of 
Table 4 Hazard ratios for overall survival

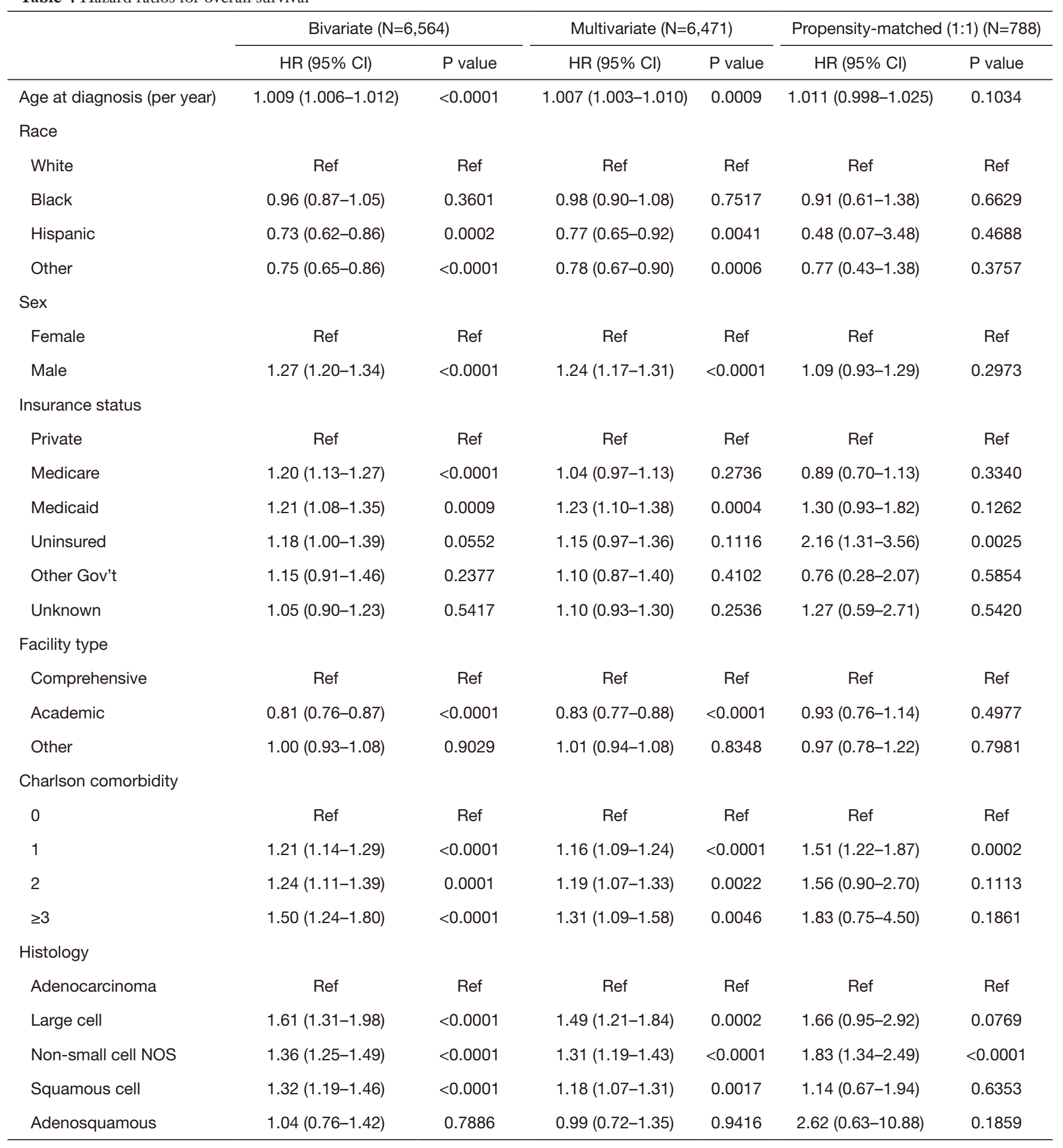

Table 4 (continued) 
Table 4 (continued)

\begin{tabular}{|c|c|c|c|c|c|c|}
\hline & \multicolumn{2}{|c|}{ Bivariate $(\mathrm{N}=6,564)$} & \multicolumn{2}{|c|}{ Multivariate $(\mathrm{N}=6,471)$} & \multicolumn{2}{|c|}{ Propensity-matched (1:1) $(\mathrm{N}=788)$} \\
\hline \multicolumn{6}{|c|}{ Number of organs with metastases } & . \\
\hline 0 & Ref & Ref & Ref & Ref & Ref & Ref \\
\hline 1 & $0.88(0.83-0.93)$ & $<0.0001$ & $0.90(0.84-0.96)$ & 0.0020 & $1.04(0.85-1.27)$ & 0.6844 \\
\hline 3 & $1.24(1.06-1.45)$ & 0.0060 & $1.41(1.20-1.65)$ & $<0.0001$ & $0.77(0.37-1.60)$ & 0.4806 \\
\hline 4 & $1.03(0.63-1.68)$ & 0.9184 & $1.07(0.64-1.78)$ & 0.8046 & N/A & $\mathrm{N} / \mathrm{A}$ \\
\hline \multicolumn{6}{|l|}{ Chemotherapy } & . \\
\hline No & Ref & Ref & Ref & Ref & Ref & Ref \\
\hline No RT & Ref & Ref & Ref & Ref & Ref & Ref \\
\hline$<39$ & $1.63(1.43-1.86)$ & $<0.0001$ & $1.67(1.46-1.91)$ & $<0.0001$ & $\mathrm{~N} / \mathrm{A}$ & $\mathrm{N} / \mathrm{A}$ \\
\hline 39 & $1.24(1.14-1.34)$ & $<0.0001$ & $1.20(1.11-1.30)$ & $<0.0001$ & $N / A$ & $N / A$ \\
\hline$>39$ & $1.05(0.96-1.14)$ & 0.2974 & $0.99(0.91-1.08)$ & 0.8553 & $0.89(0.76-1.05)$ & 0.1526 \\
\hline SBRT & $0.58(0.43-0.78)$ & 0.0003 & $0.60(0.44-0.81)$ & 0.0009 & $\mathrm{~N} / \mathrm{A}$ & $N / A$ \\
\hline Year of diagnosis & $0.98(0.97-0.99)$ & $<0.0001$ & $0.98(0.97-0.99)$ & 0.0005 & $1.00(0.96-1.04)$ & 0.9452 \\
\hline
\end{tabular}

the PACIFIC study found that the PFS improvement in favor of durvalumab was more pronounced in patients who had their last radiation dose within two weeks of starting IT compared to those who had their last radiation dose earlier (12). Few studies have tested concomitant administration of RT + IT, and only a few case reports have described a benefit with IT before RT (14,35-37). This study also pointed to a possible interaction effect between BED and sequencing of RT + IT, with patients who received lower BED showing improved OS with RT prior to eIT compared to those that received RT after eIT, even after conducting a sensitivity analysis that restricted the timing difference between eIT and RT to 30 days. Earlyonset disease progression may play an important role in this relationship, as progression on eIT requiring palliative RT often portends poor prognosis.

The interactions of radiation and immunotherapy seen in our analysis may be influenced by many other factors including the number of metastases present. Two recent randomized trials have shown that local consolidative therapy is associated with improved OS in patients with oligometastatic NSCLC after initial treatment. Gomez et al. reported that local consolidative therapy (including conventional RT, SBRT, or surgery) in patients with oligometastatic NSCLC that did not progress after frontline therapy was associated with a significantly improved PFS (median, 14.2 months) and OS (median, 41.2 months) compared to maintenance therapy or palliative care (38-40). Similarly, SABR-COMET also found that SBRT in oligometastatic patients was associated with improved OS (median, 41 months) compared to those without SBRT (median, 28 months) with no impact on quality of life $(41,42)$. While these studies point to potential impact of number of metastases, we were not able to stratify for this within our analysis and look forward to the results of ongoing trials, including SABR COMET-10, to shed light on this interaction.

Additionally, while some have demonstrated "immunogenic" effects of SBRT at an ablative dose, there is lack of clarity in these studies as to what specific radiation schedules, dosing, and fractionations result in the most optimal immune response necessary to improve 
OS and PFS (34). To date there have been promising data suggesting that in PD-L1 negative tumors, the addition of SBRT to immunotherapy can improve numerical survival. Additionally, Bauml et al. found promising outcomes with SBRT was integrated with immunotherapy in oligometastatic NSCLC patients (43). It is possible that the non-ablative (palliative) SBRT doses in this study may explain the lack of OS or PFS benefit in relation to eIT in the present analysis.

The key strength of this study is the large number of mNSCLC patients identified using a large, national, hospital-based cancer registry. However, these results are subject to the limits of a retrospective database review including lack of centralized pathological review, lack of standardization in RT techniques, and selection bias in the treatment modalities offered. Additionally, performance status, a crucial prognostic factor in clinical trial eligibility and cancer treatment decision-making, is not available. There is also no information on smoking history, molecular genotype (KRAS, EGFR, ALK, etc.), or PD-L1 analysis of the tumor in NCDB. In particular, molecular markers and mutational burden of lung cancer tumors are critical indicators of the effectiveness of eIT in these patients (44). Imbalances in the biomarkers may be contributing to differential OS outcomes in this analysis. Multivariate and propensity score matched analyses were conducted to minimize these risks, yet the impact of unmeasured confounders cannot be fully mitigated. In addition, the NCDB does not collect data on locoregional or distant recurrence, cause-specific survival, or treatment-related toxicities, endpoints that would be useful in understanding the full impact of a combined modality approach.

\section{Conclusions}

The utilization of RT in combination with eIT is increasing. The use of any RT in the context of eIT was associated with inferior OS, likely as a result of patient selection and imbalance of prognostic factors. The hypothesis-generating observation of superior OS for patients receiving SBRT and the interaction between BED and RT + eIT sequencing suggest that RT may provide immunomodulatory benefits to a select group of patients. These results underscore the need for more prospective clinical trials examining radiation dose, sequencing, and site in multi-modality treatment and support efforts to evaluate optimal radiation modality, doses, and sequencing patterns in prospective randomized trials.

\section{Acknowledgments}

Funding: None.

\section{Footnote}

Reporting Checklist: The authors have completed the STROBE reporting checklist. Available at http://dx. doi. org/10.21037/tlcr-20-537

Conflicts of Interest: All authors have completed the ICMJE uniform disclosure form (available at http://dx. doi. org/10.21037/tlcr-20-537). Dr. NER reports personal fees from BMS, personal fees from Celgene, grants and personal fees from Merck, personal fees from Astrazeneca, personal fees from G1 therapeutics, personal fees from Novartis, personal fees from Genentech, personal fees from Roche, personal fees from Abbvie, outside the submitted work. Dr. JMC reports grants from Bristol-Myers Squibb, grants from Genentech, grants from Spectrum, grants from Adaptimmune, grants from Medpacto, grants from Bayer, grants from Abbvie, grants from Moderna, grants from Array, grants and personal fees from Eli Lilly, personal fees from Merck, personal fees from AstraZeneca, personal fees from Guardant Merck, personal fees from Achilles Therapeutics, outside the submitted work. The other authors have no conflicts of interest to declare.

Disclaimer: The data used in the study are derived from a deidentified NCDB file. The American College of Surgeons and the Commission on Cancer have not verified and are not responsible for the analytic or statistical methodology employed, or the conclusions drawn from these data by the investigator.

Ethical Statement: The authors are accountable for all aspects of the work in ensuring that questions related to the accuracy or integrity of any part of the work are appropriately investigated and resolved. All procedures performed in this study were in accordance with the Declaration of Helsinki (as revised in 2013). Because of the retrospective nature of the research, the requirement for informed consent was waived.

Open Access Statement: This is an Open Access article distributed in accordance with the Creative Commons Attribution-NonCommercial-NoDerivs 4.0 International License (CC BY-NC-ND 4.0), which permits the non- 
commercial replication and distribution of the article with the strict proviso that no changes or edits are made and the original work is properly cited (including links to both the formal publication through the relevant DOI and the license). See: https://creativecommons.org/licenses/by-nc-nd/4.0/.

\section{References}

1. Cancer Facts \& Figures 2019. Atlanta: American Cancer Society; 2019.

2. Herbst RS, Morgensztern D, Boshoff C. The biology and management of non-small cell lung cancer. Nature 2018;553:446.

3. Gandhi L, Garassino MC. Pembrolizumab plus Chemotherapy in Lung Cancer. N Engl J Med 2018;379:e18.

4. Pai-Scherf L, Blumenthal GM, Li H, et al. FDA Approval Summary: Pembrolizumab for Treatment of Metastatic Non-Small Cell Lung Cancer: First-Line Therapy and Beyond. Oncologist 2017;22:1392-9.

5. Goldberg SB, Gettinger SN, Mahajan A, et al. Pembrolizumab for patients with melanoma or non-smallcell lung cancer and untreated brain metastases: early analysis of a non-randomised, open-label, phase 2 trial. Lancet Oncol 2016;17:976-83.

6. Weichselbaum RR, Liang H, Deng L, et al. Radiotherapy and immunotherapy: a beneficial liaison? Nat Rev Clin Oncol 2017;14:365.

7. Ko EC, Raben D, Formenti SC. The Integration of Radiotherapy with Immunotherapy for the Treatment of Non-Small Cell Lung Cancer. Clin Cancer Res 2018;24:5792-806.

8. Pirker R, Pereira JR, Szczesna A, et al. Prognostic factors in patients with advanced non-small cell lung cancer: data from the phase III FLEX study. Lung Cancer 2012;77:376-82 .

9. Brundage MD, Davies D, Mackillop WJ. Prognostic factors in non-small cell lung cancer: a decade of progress. Chest 2002;122:1037-57.

10. Machtay M, Bae K, Movsas B, et al. Higher biologically effective dose of radiotherapy is associated with improved outcomes for locally advanced non-small cell lung carcinoma treated with chemoradiation: an analysis of the Radiation Therapy Oncology Group. Int J Radiat Oncol Biol Phys 2012;82:425-34.

11. Shaverdian N, Lisberg AE, Bornazyan K, et al. Previous radiotherapy and the clinical activity and toxicity of pembrolizumab in the treatment of non-small-cell lung cancer: a secondary analysis of the KEYNOTE-001 phase
1 trial. Lancet Oncol 2017;18:895-903.

12. Antonia SJ, Villegas A, Daniel D, et al. Durvalumab after Chemoradiotherapy in Stage III Non-Small-Cell Lung Cancer. N Engl J Med 2017;377:1919-29.

13. Verma V, Cushman TR, Selek U, et al. Safety of Combined Immunotherapy and Thoracic Radiation Therapy: Analysis of 3 Single-Institutional Phase I/II Trials. Int J Radiat Oncol Biol Phys 2018;101:1141-8.

14. Boyer MJ, Gu L, Wang X, et al. Toxicity of definitive and post-operative radiation following ipilimumab in nonsmall cell lung cancer. Lung Cancer 2016;98:76-8.

15. Theelen WSME, Peulen HMU, Lalezari F, et al. Effect of Pembrolizumab After Stereotactic Body Radiotherapy vs Pembrolizumab Alone on Tumor Response in Patients With Advanced Non-Small Cell Lung Cancer: Results of the PEMBRO-RT Phase 2 Randomized Clinical Trial Pembrolizumab Alone vs After Stereotactic Body Radiotherapy in Patients With Advanced NSCLCPembrolizumab Alone vs After Stereotactic Body Radiotherapy in Patients With Advanced NSCLC. JAMA Oncol 2019;5:1276-82.

16. Foster CC, Sher DJ, Rusthoven CG, et al. Overall survival according to immunotherapy and radiation treatment for metastatic non-small-cell lung cancer: a National Cancer Database analysis. Radiat Oncol 2019;14:18.

17. Bilimoria KY, Stewart AK, Winchester DP, et al. The National Cancer Data Base: a powerful initiative to improve cancer care in the United States. Ann Surg Oncol 2008;15:683-90.

18. Mohanty S, Bilimoria KY. Comparing national cancer registries: The National Cancer Data Base (NCDB) and the Surveillance, Epidemiology, and End Results (SEER) program. J Surg Oncol 2014;109:629-30.

19. Goldstraw P, Chansky K, Crowley J, et al. The IASLC Lung Cancer Staging Project: Proposals for Revision of the TNM Stage Groupings in the Forthcoming (Eighth) Edition of the TNM Classification for Lung Cancer. J Thorac Oncol 2016;11:39-51.

20. Fowler JF. 21 years of biologically effective dose. Br J Radiol 2010;83:554-68.

21. Chi A, Wen S, Liao Z, et al. What would be the most appropriate alpha/beta ratio in the setting of stereotactic body radiation therapy for early stage non-small cell lung cancer. BioMed Res Int 2013;2013:391021.

22. Rodrigues G, Videtic GMM, Sur R, et al. Palliative thoracic radiotherapy in lung cancer: An American Society for Radiation Oncology evidence-based clinical practice guideline. Pract Radiat Oncol 2011;1:60-71. 
23. Lutz S, Balboni T, Jones J, et al. Palliative radiation therapy for bone metastases: Update of an ASTRO Evidence-Based Guideline. Pract Radiat Oncol 2017;7:4-12.

24. Jacobs CD, Carpenter DJ, Hong JC, et al. Radiation Records in the National Cancer Database: Variations in Coding and/or Practice Can Significantly Alter Survival Results. JCO Clin Cancer Inform 2019;3:1-9.

25. Tipton KN, Sullivan N, Bruening W, et al. Stereotactic Body Radiation Therapy. Rockville (MD): Agency for Healthcare Research and Quality (US); 2011.

26. Deyo RA, Cherkin DC, Ciol MA. Adapting a clinical comorbidity index for use with ICD-9-CM administrative databases. J Clin Epidemiol 1992;45:613-9.

27. Torok JA, Gu L, Tandberg DJ, et al. Patterns of Distant Metastases After Surgical Management of Non-Small-cell Lung Cancer. Clin Lung Cancer 2017;18:e57-70.

28. Hasselle MD, Haraf DJ, Rusthoven KE, et al. Hypofractionated image-guided radiation therapy for patients with limited volume metastatic non-small cell lung cancer. J Thorac Oncol 2012;7:376-81.

29. Hwang WL, Niemierko A, Hwang KL, et al. Clinical Outcomes in Patients With Metastatic Lung Cancer Treated With PD-1/PD-L1 Inhibitors and Thoracic Radiotherapy. JAMA Oncol 2018;4:253-5.

30. Theelen WSME, Peulen HMU, Lalezari F, et al. Randomized phase II study of pembrolizumab after stereotactic body radiotherapy (SBRT) versus pembrolizumab alone in patients with advanced non-small cell lung cancer: The PEMBRO-RT study. J Clin Oncol 2018;36:9023.

31. Wang YS, Yang G, Wang YY, et al. Early efficacy of stereotactic body radiation therapy combined with adoptive immunotherapy for advanced malignancies. Mol Clin Oncol 2013;1:925-9.

32. Luke JJ, Lemons JM, Karrison TG, et al. Safety and Clinical Activity of Pembrolizumab and Multisite Stereotactic Body Radiotherapy in Patients With Advanced Solid Tumors. J Clin Oncol 2018;36:1611-8.

33. Twyman-Saint Victor C, Rech AJ, Maity A, et al. Radiation and dual checkpoint blockade activate non-redundant immune mechanisms in cancer. Nature 2015;520:373-7.

34. Mazzola R, Jereczek-Fossa BA, Franceschini D, et al. Oligometastasis and local ablation in the era of systemic targeted and immunotherapy. Radiat Oncol 2020;15:92.

35. Golden EB, Chhabra A, Chachoua A, et al. Local radiotherapy and granulocyte-macrophage colonystimulating factor to generate abscopal responses in patients with metastatic solid tumours: a proof-of-principle trial. Lancet Oncol 2015;16:795-803.

36. Tang C, Welsh JW, de Groot P, et al. Ipilimumab with Stereotactic Ablative Radiation Therapy: Phase I Results and Immunologic Correlates from Peripheral T Cells. Clin Cancer Res 2017;23:1388-96.

37. Komatsu T, Nakamura K, Kawase A. Abscopal Effect of Nivolumab in a Patient with Primary Lung Cancer. J Thorac Oncol 2017;12:e143-4.

38. Gomez DR, Blumenschein GR Jr, Lee JJ, et al. Local consolidative therapy versus maintenance therapy or observation for patients with oligometastatic non-smallcell lung cancer without progression after first-line systemic therapy: a multicentre, randomised, controlled, phase 2 study. Lancet Oncol 2016;17:1672-82.

39. Gomez DR, Tang C, Zhang J, et al. Local Consolidative Therapy Vs. Maintenance Therapy or Observation for Patients With Oligometastatic Non-Small-Cell Lung Cancer: Long-Term Results of a Multi-Institutional, Phase II, Randomized Study. J Clin Oncol 2019;37:1558-65.

40. Iyengar P, Wardak Z, Gerber DE, et al. Consolidative Radiotherapy for Limited Metastatic Non-Small-Cell Lung Cancer: A Phase 2 Randomized Clinical Trial. JAMA Oncol 2018;4:e173501.

41. Palma DA, Olson R, Harrow S, Get al. Stereotactic ablative radiotherapy versus standard of care palliative treatment in patients with oligometastatic cancers (SABRCOMET): a randomised, phase 2, open-label trial. Lancet 2019;393:2051-8.

42. Palma DA, Olson R, Harrow S, et al. Stereotactic Ablative Radiotherapy for the Comprehensive Treatment of Oligometastatic Cancers: Long-Term Results of the SABR-COMET Phase II Randomized Trial. J Clin Oncol 2020;38:2830-8.

43. Bauml JM, Mick R, Ciunci C, et al. Pembrolizumab After Completion of Locally Ablative Therapy for Oligometastatic Non-Small Cell Lung Cancer: A Phase 2 Trial. JAMA Oncol 2019;5:1283-90.

44. Sidransky D. Emerging molecular markers of cancer. Nat Rev Cancer 2002;2:210-9.

Cite this article as: Chodavadia PA, Jacobs CD, Wang F, Salama JK, Kelsey CR, Clarke JM, Ready NE, Torok JA. Synergy between early-incorporation immunotherapy and extracranial radiotherapy in metastatic non-small cell lung cancer. Transl Lung Cancer Res 2021;10(1):261-273. doi: 10.21037/tlcr-20-537 